\title{
Deduction through the Ages: A History of Truth
}

\author{
Jerry Lodder*
}

\section{Introduction}

While the subtitle "A History of Truth" is far too ambitious for this brief slice through the history of selected topics in deductive thought, this project does offer, in the words of the pioneers, a few passages that explain how modern mathematics has arrived at an understanding of elementary propositional logic. Different cultures have held differing views about deduction, i.e. whether a conclusion can be drawn from certain given information, and whether the conclusion is absolute or relative. Consider the following two examples of conditional propositions, each phrased in terms of the words "if . . , then . . : :"

1. If a person is born at the rising of the Dog Star, then that person will not die at sea.

2. If $n$ is an odd integer, then $\left(n^{2}-1\right) / 4$ is an even integer.

The first was hypothesized by the ancient Greeks, while the second is a statement from modern mathematics. Both are examples of what today are called "implications" ("if-then" statements), although the first is of a speculative nature, useful for its predictive power, and the second is rather rigid. Given an odd integer $n$, then either $\left(n^{2}-1\right) / 4$ is an even integer or not, decidable immediately without speculation about future events. The ancient Greeks likely held a more fluid view of logic than we do today.

Presented in this project are a few excerpts from the works of some of the most influential philosophers, mathematicians and logicians over two millennia who have contributed to the modern interpretation of the truth of an implication. In this abbreviated account, justice can not be done to the various nuanced intellectual and cultural settings in which each author was writing. However, the reader may glean a more balanced and comprehensive overview of deductive thought than that offered by a purely formulaic treatment found in many textbooks.

The project opens with a brief passage from the ancient Greek philosopher Aristotle (384-322 B.C.E), whose work in philosophy, politics, logic and the natural sciences has survived until today. Of importance here is a term, often translated as a "syllogism," which according to Aristotle "is speech in which, certain things having been supposed, something different from those supposed results of necessity because of their being so" $[1$, p. 66] [26]. In the sequel, "syllogism" will denote any rule of reasoning, without restriction to a particular form. The types of syllogisms used by Aristotle often apply to categories of objects, such as the following: All humans are mortal. All mortals will die. Therefore, all humans will die. Aristotle's logic, however, appears not to have been used by Greek mathematicians in antiquity, who opted for other rules of deduction.

\footnotetext{
*Mathematical Sciences; Dept. 3MB, Box 30001; New Mexico State University; Las Cruces, NM 88003; jlodder@nmsu.edu.
} 
The particular syllogisms forming the core of the project are those attributed to the lesserknown Greek philosopher Chrysippus (280-206 B.C.E.), whose work in logic rivaled that of Aristotle. Presented are five rules of inference, called indemonstrable argument forms, which involve the terms "and," "or," "not," and "if-then." Since no known complete work of Chrysippus remains today, we must rely on fragments, collected by secondary authors. A central plot line of the project, moving well beyond the work of those in antiquity, is whether these five rules attributed to Chrysippus are special cases of just one rule, and, if not, which rules are logically the same. Verbal argument asserting the equivalence of certain rules is difficult, and a more streamlined method for discussing their relation to each other is sought.

In the modern era, an initial attempt at a symbolic and almost calculational form of elementary logic is examined from the work of the English mathematician George Boole (1815-1864). Author of An Investigation of the Laws of Thought [5], Boole believed that he had reduced deductive thought to a system of calculation involving the signs $\times,+,-$, borrowed from arithmetic. Quoted here are only are a few brief passages from Boole's monumental treatise [6], designed to introduce the reader to how statements involving terms like "and," "or," and "not" could even be written using the $\times,+,-$ notation. What verbal meanings do these symbols actually capture? Do the symbols retain their algebraic meaning, or are they subject to non-algebraic rules, since they express "the laws of thought" ? An important outgrowth of Boole's "algebra of statements" is the convention of assigning true statements the value 1 , and false statements the value 0 .

The German mathematician and philosopher Gottlob Frege (1848-1925) introduced a radically new system of notation for logic, called a concept-script or "Begriffsschrift" in the original German, which did not use Boole's $\times,+,-$ notation. Within the concept-script, Frege uses a special symbol, called the condition stroke ${ }^{1}$ that carries a truth value recognized today as being very close to that in Chrysippus's first two rule of inference. In fact, the progression of thought from antiquity to today is quite compelling. The ancient Greek philosopher Philo of Megara (ca. 4th century B.C.E.) maintained that a valid hypothetical proposition is "that which does not begin with a truth and end with a falsehood" [23, II. 110], while Frege maintained that the symbol $B$ is true except A

when $A$ is true and $B$ is false, a comparison to be explored in Exercises 5.9 and 6.2. Again, the intellectual and cultural settings in which these two statements were written are relevant.

While Frege successfully established a break between statements in mathematics and the logic needed to manipulate those statements, his notation is a bit awkward. His work is codified and greatly expanded in the lucid prose of the English mathematicians and philosophers Bertrand Russell (1872-1970) and Alfred North Whitehead (1861-1947), who set the stage for mathematical logic in the twentieth century. Both Englishmen were practitioners of logicism, the belief that mathematical truths could be deduced from truths in logic. In their magnum opus Principia Mathematica [21], a streamlined notation for logic different from that of Frege and Boole is introduced. The condition stroke of Frege is replaced with a more convenient symbol. In fact, all five rules attributed to Chrysippus can be succinctly rewritten in the notation of Russell and Whitehead, an important first step in deciding the logical equivalence of these rules. Additionally Russell was a prolific writer, contributing to the fields of education, history, religion, and political theory. He was an advocate of social reform, an anti-war activist, and later in life an anti-nuclear activist. His anti-war protests in England resulted in his dismissal from Trinity College in 1916, and a five-month imprisonment in 1918. 
In Principia Mathematica Russell and Whitehead state that the truth-values of statements involving terms like "and," "or," "not" depend only upon the elementary propositions to which the these terms apply. Exactly how is the truth value of a complicated statement determined from its elementary pieces? One solution to this is offered by the Austrian logician Ludwig Wittgenstein (1889-1951) whose "schemata" provide a simple overview, in tabular format, for the truth of a complicated (compound) statement. All five rules attributed to Chrysippus can be written using these schemata, and then compared among themselves. Wittgenstein introduces the term "truth grounds" for those values ("true" or "false") of the elementary propositions that yield the truth of a compound statement built from its elementary pieces. This he uses to state the conditions under which an implication (an "if-then" statement) is true, which is applicable to Chrysippus's first two rules of inference.

Independently of Wittgenstein, Emil Post (1897-1954) developed a nearly identical tabular format for displaying the truth values of a compound statement, and he dubbed these displays "truth tables," the name in current use today. Emil was born in Poland, of Jewish parents, with whom he emigrated to New York in 1904. He received his doctorate from Columbia University, where he participated in a seminar devoted to the study of Principia Mathematica. In his dissertation of 1921, "Introduction to a General Theory of Propositional Functions" [18], he develops the notion of truth tables and clearly displays the table for an implication. With this in hand, a simple analysis of all five rules attributed to Chrysippus follows. Sadly, Emil suffered from manic depression, a condition treated by electro-convulsive shock. His death from a sudden heart attack occurred in a mental institution shortly after one these treatments [19, p. xii].

Exercise 1.1. In what constellation does the Dog Star appear? By what name is the Dog Star known today?

Exercise 1.2. For each of the odd integers $n=1,3,5$, determine whether $\left(n^{2}-1\right) / 4$ is an even integer. Can you prove in general that if $n$ is any odd integer, then $\left(n^{2}-1\right) / 4$ is an even integer?

\section{Aristotle Defines Syllogism}

A towering figure in the history of deductive thought is the ancient Greek philosopher and generalist Aristotle (384-322 B.C.E.), whose works in logic, government, and natural science have been studied and revisited ever since their authorship more than two millennia ago. The young Aristotle studied at Plato's Academy in Athens until Plato's death in 347. After this Aristotle tutored Alexander the Great at the court of Alexander's father, Philip II of Macedon. Eventually Aristotle returned to Athens, where he founded his own school, the Lyceum [16, p. 54]. He contributed to virtually all fields of knowledge, particularly the sciences, such as biology, physics and astronomy, where his work as an empiricist (observer and recorder of facts) is preeminent [10]. Let's turn to his work in logic, particularly the use of deduction, which may have been an outgrowth of his inquiry into the scientific method. Although this project will examine in detail the work of other philosophers, some more relevant to the type of deductive reasoning presented here, we begin with Aristotle, whose work was transmitted to the Arabic and Latin medieval arenas, from which it greatly influenced European and present-day thought. Aristotle acknowledges the role that language must play in a study of logic and limits his consideration to statements that are true or false. A brief excerpt from his writing On Interpretation [1, p. 42-45] [2] reads:

\section{0}

Every sentence has meaning, not as being the natural means by which a physical faculty is realized, but, ... by convention. Yet every sentence is not a proposition; only such are propositions as have in them either truth or falsity. Thus, a prayer is a sentence, but is neither true nor false. 
Let us therefore dismiss all other types of sentences but the proposition, for this last concerns our present inquiry .... Every proposition must contain a verb or the tense of a verb. ...

In the case of that which is or which has taken place, propositions ... must be true or false.

20000000000

Exercise 2.1. Based on Aristotle's description, which of the following are propositions? Justify your answer. If you have difficulty with a particular item, explain what the difficulty is.

(a) Let there be light.

(b) The number three.

(c) A square has five sides.

(d) Every coyote has four legs.

(e) This sentence is false.

Aristotle begins Prior Analytics with "We must first state the subject of our inquiry and the faculty to which it belongs: its subject is demonstration and the faculty that carries it out demonstrative science" [1, p. 65] [3]. A key tool in the demonstration, i.e., verification, of a proposition is what has become known as a syllogism. "A deduction [syllogism] is speech in which, certain things having been supposed, something different from those supposed results of necessity because of their being so" $[1$, p. 66] [26]. Aristotle discusses several types of syllogisms or rules of inference, beginning with the "first figure" of term arrangements: "If $A$ is predicated of all $B$, and $B$ of all $C, A$ must be predicated of all $C$ " $[1$, p. 68]. We will call this "Aristotle's first figure," while $A$, $B$ and $C$ are called terms. A property or quality $A$ predicated of all $B$ means that every $B$ has property $A$, or every $B$ is an $A$. Let's examine the use of this syllogism.

Exercise 2.2. Identify the terms $A, B$ and $C$ in the first figure for the statement: "Having four sides is predicated of every rectangle, and being a rectangle is predicated of every square." What conclusion can be drawn from these premises? How can the statement " $A$ is predicated of every $B$ " be written more directly beginning with "Every $B \ldots$ "? How can " $B$ is predicated of every $C$ " be written more directly beginning with "Every $C \ldots$. ? Find a more direct statement (in the active voice) of Aristotle's first figure using these simplifications. Rewrite "Having four sides is predicated of every rectangle, and being a rectangle is predicated of every square" using this reformulation.

Exercise 2.3. Let $A, B$ and $C$ be terms in the sense of Aristotle. Consider the statements:

(I) If $C$ occurs, then $B$ occurs; and if $B$ occurs, then $A$ occurs.

(II) If $C$ occurs, then $A$ occurs.

Would you claim that (II) is a logical inference from (I)? If so, support your position using the work of Aristotle. If not, find a counter example. Would you claim that (I) is a logical inference from (II)? If so, support your position using the work of Aristotle. If not, find a counter example. 


\section{Chrysippus and Five Indemonstrable Forms}

While studied extensively, Aristotle's term logic appears not to have been used by ancient Greek mathematicians [16, p. 55], including Euclid. How would the reader prove the statement "the base angles of an isosceles triangle are congruent" by concatenation of terms? A rival logic to that of Aristotle arose in the Hellenistic period (ca. late third century through the first century B.C.E), namely that of the Stoic philosophers, who maintained that enlightenment arose from emotionally detached judgments. During its time, Stoic logic was held in higher regard than Aristotle's logic [26], although no known complete works of the Stoics survive today, with only fragments remaining. Chief among the Stoics was Chrysippus (ca. 280-206 B.C.E.) of whom Diogenes Laertius some 500 years later wrote "So renowned was he [Chrysippus] for dialectic that most people thought, if the gods took to dialectic, they would adopt no other system than that of Chrysippus" [7, p. 289]. Writing in Greek, the commentator Diogenes Laertius (ca. third century C.E.) continues [7, p. 187]

Further, arguments may be divided into true and false. The former draw their conclusions by means of true premisses .... Those are false which have error in the premisses or are inconclusive .... Further, there are statements which are indemonstrable, because they do not need demonstration; they are employed in the construction of every argument. As to the number of these, authorities differ; Chrysippus makes them five.

Let's examine Chrysippus's five argument forms attributed to Chrysippus, although other forms were in use. Since his works are no longer extant, we must rely on fragments collected by secondary authors [7, p. 189] [13, p. 83] [17, p. 212-213].

\section{0}

1. If the first, [then] the second. The first. Therefore, the second.

2. If the first, [then] the second. Not the second. Therefore, not the first.

3. Not both the first and the second. The first. Therefore, not the second.

4. Either the first or the second. The first. Therefore, not the second.

5. Either the first or the second. Not the first. Therefore, the second.

\section{0}

Examples of these rules of inference abound, but let's consider those given in Diogenes Laertius and by others in antiquity [13, p. 84]. Note that (curiously) no example of the fourth rule is provided in these fragments.

1. If it is day, there is light. It is day. Therefore, there is light.

2. If it is day, there is light. There is not light. Therefore, it is not day.

3. It is not the case both that it is day and it is night. It is day. Therefore, it is not night.

5. Either it is day or it is night. It is not night. Therefore, it is day. 
In each of the syllogisms above, a conclusion is being drawn from two given statements, the first a more general statement, e.g., "Either it is day or it is night," and the second a more specific statement, e.g., "It is not night," with the conclusion in this case being "It is day." The first, more general statement, is often called the major premise, while the second, more specific statement, is called the minor premise. The five rules of inference (syllogisms) above all begin with a major premise, followed by a minor premise and a conclusion. The major premise of the first two rules are examples of "if-then" statements, also commonly called "hypothetical propositions" in translation from ancient Greek [24, II. 111-113]. The truth of a hypothetical proposition itself was a matter of debate in antiquity [12]. Within the structure of an "if-then" statement, the "if" clause is called the antecedent, while the "then" clause is called the consequent. The Greek physician, philosopher, and commentator Sextus Empiricus (ca. 160-210 C.E.) states [24, II. 112]: "Now all the Dialecticians agree in asserting that a hypothetical proposition is valid when its consequent follows [from] its antecedent, but as to when and how it so follows they disagree with one another, and propound conflicting criteria of this 'following.' " Another philosopher of antiquity, Philo of Megara, (ca. 4th century B.C.E.) maintained that a valid hypothetical proposition is "that which does not begin with a truth and end with a falsehood" [23, II. 110], a statement studied further in Exercise 5.9. An issue in understanding these passages may be in the translation and interpretation of ancient Greek, while another problem lies in a confusion between modern and ancient meanings of terms like "hypothetical proposition," "antecedent," and "consequent."

Let's move to the other indemonstrable forms, and examine their major premises. The third has as major premise "Not both the first and the second," while the fourth and fifth rules both begin with "Either the first or the second." Could a major premise involving an "and" statement be logically the same as a major premise involving an "or" statement? Are the two major premises involving "or" actually equivalent? Why are two rules stated for the use of "or"?

Exercise 3.1. Although we cannot be certain of the logical import of the connective "or" as used by Diogenes Laertius in the interpretation of Chrysippus, consider two common uses of "or" in language today:

(I) For $\$ 7.95$ the meal comes with either soup or salad.

(II) As a graduation requirement in science, a student must pass either a physics course or a chemistry course.

(a) In statement (I) is the conclusion valid, namely that the meal costs $\$ 7.95$, if both conditions of the "or" statement are met, i.e., both soup and salad are ordered?

(b) In statement (II) is the conclusion valid, namely that a student fulfills a graduation requirement in science, if both conditions of the "or" statement are met, namely the student passes both a course in physics and a course in chemistry?

(c) Today we say that the statement " $A$ or $B$ " represents the exclusive or if the truth of " $A$ or $B$ " excludes the possibility that both $A$ and $B$ are true. Similarly, " $A$ or $B$ " represents the inclusive or if the truth of " $A$ or $B$ " includes the possibility that both $A$ and $B$ are true. In the fourth rule of inference, Chrysippus states "Either the first or the second. The first. Therefore, not the second." Must the conclusion "Therefore not the second" necessarily follow for the "inclusive or"? the "exclusive or"? Explain your answer with a concrete example, providing actual statements for "the first" and "the second."

(d) In the example of Chrysippus's fifth rule, "Either it is day or it is night. It is not night. Therefore, it is day," is a particular type of "or" being used? Must the 
conclusion "Therefore it is day" necessarily follow for the "inclusive or"? for the "exclusive or"? Justify your answer.

(e) When Aristotle states that a proposition is either true or false, what type of "or" is being used?

Exercise 3.2. The major premise of the first rule of inference is an example of an "if-then" statement. Consider the statement: "If the roof leaks, then the house will not sell." Using the second rule of inference, what can be concluded if the house will sell? Write this as an "if-then" statement beginning with "If the house will sell, ..." Then letting $A$ and $B$ denote general propositions, rewrite "If $A$, then $B$ " as a different "if-then" statement using the pattern of the second rule of inference.

Exercise 3.3. The major premise of the third rule of inference is the negation (not the case) of an "and" statement. Rewrite "It is not the case both that it is day and it is night" as an "if-then" statement so that when the pattern of the first rule of inference is followed, the minor premise becomes "It is day," and the conclusion matches that in the example for the third rule of inference. Then, for elementary propositions $A$ and $B$, rewrite "It is not the case that both $A$ and $B$ " as an "if-then" statement following this pattern.

Exercise 3.4. The major premise of the fourth rule of inference is an "exclusive or" statement. Suppose that "either $A$ or $B$, but not both" is given and that $A$ is true. What can be concluded about $B$ ? Justify your answer.

Exercise 3.5. For the inclusive or, write "either $A$ or $B$ " as an "if-then" statement following the pattern of the fifth rule of inference.

Exercise 3.6. Speculate on whether every "if-then" statement can be written as an "and" statement. Speculate on whether every "if-then" statement can be written as an "exclusive or" statement, or as an "inclusive or" statement. Apply your conjectures to the statement "if the roof leaks, then the house will not sell." At the end of the project in Exercise 8.8 we will return to this question.

\section{Boole's Algebra of Statements}

An old point of view on logic is to reduce the subject to a system of calculation, whereby the rules of reasoning could be automated. The German philosopher, mathematician, and universalist Gottfried Wilhelm Leibniz (1646-1716) was one of the first to pursue this idea, and sought a characteristica generalis (general characteristic) or a lingua generalis (general language) that would serve as a universal symbolic language and reduce all debate to calculation. Leibniz maintained "If controversies were to arise, there would be no more need of disputation between two philosophers than between two accountants. For it would suffice to take their pencils in their hands, to sit down to their slates, and to say to each other: ... Let us calculate" [22, p. 170]. What form should a universal language assume? What system of calculation is best suited for computations in reasoning? The solution to such a query is framed by existing systems of calculations.

The self-taught English mathematician George Boole (1815-1864) begins his 1854 opus An Investigation of the Laws of Thought [5] with "The design of the following treatise is to investigate the fundamental laws of those operations of the mind by which reasoning is performed; to give expression to them in the symbolical language of a calculus ${ }^{2}$, and upon this foundation to establish

\footnotetext{
${ }^{2}$ a system of calculation
} 
the science of Logic and construct its method" [6, p. 1]. The symbols chosen by Boole to represent the fundamental laws of reasoning are borrowed from algebra, namely $\times,+,-$, although their meaning is not in an algebraic sense, but a logical one. Boole actually believed that he had captured "the laws of thought," and a detailed study of his treatise [6] reveals the calculational scope of his methods. Presented here are only a few passages designed to indicate how terms like "and," "or," "except" are rewritten using the symbols $\times,+,-$. Boole's use of "or" is in the exclusive sense, but in the exercises, "or" is often inclusive. Although at the time the work was not applied to the design of automated calculating machines, today the ideas introduced in The Laws of Thought are seen as fundamental to the construction of certain circuits in digital computers, and a "Boolean variable" is one which assumes only one of two possible values, 1 or 0 , "true" or "false." For further material on this topic, see Janet Barnett's projects on Boolean algebra.

Boole was originally a school teacher, but in 1849 was appointed as a founding Professor of Mathematics at Queen's College in Cork, Ireland. Apparently he was unaware of Leibniz's goal to find a universal symbolic language as The Laws of Thought was in press [11, p. 51]. Let's read a few excerpts from this treatise. [6, p. 27-48].

\section{0}

\section{Proposition I.}

All the operations of Language, as an instrument of reasoning, may be conducted by a system of signs composed of the following elements, viz:

1st. Literal symbols, as $x, y \& c$. , representing things as subjects of our conceptions.

2nd. Signs of operation, as,,$+- \times$, standing for those operations of the mind by which the conceptions of things are combined or resolved so as to form new conceptions involving the elements.

3rd. The sign of identity, $=$.

And these symbols of Logic are in their use subject to definite laws, partly agreeing with and partly differing from the laws of the corresponding symbols in the science of Algebra.

Let it be assumed as a criterion of the true elements of rational discourse, that they should be susceptible of combination in the simplest forms and by the simplest laws, and thus combining should generate all other known and conceivable forms of language; and adopting this principle, let the following classification be considered.

\section{CLASS I.}

Appellative or descriptive signs, expressing either the name of a thing or some quality or circumstance belonging to it.

To this class we may obviously refer the substantive ${ }^{3}$ proper or common, and the adjective. ...

Let us then agree to represent the class of individuals to which a particular name or description is applicable, by a single letter, as $x$. ... By a class is usually meant a collection of individuals, to each of which a particular name or description may be applied; but in this work the meaning of the term will be extended so as to include the case in which but a single individual exists, answering to the required name or description, as well as the cases denoted by the terms "nothing" and "universe," which as "classes" should be understood to comprise respectively "no beings," and "all beings." ... Let it further be agreed, that by the combination $x y$ shall be represented that class of things to which the names or descriptions represented by $x$ and $y$ are simultaneously applicable. ...

[I]f $x$ represent "estuaries," and $y$ "rivers," the expressions $x y$ and $y x$ will indifferently represent "rivers that are estuaries," or "estuaries that are rivers," ... . Let there be a third symbol, as $z$, representing that class of things to which the term "navigable" is applicable, and any one of the

\footnotetext{
${ }^{3}$ noun
} 
following expressions,

$$
z x y, \quad z y x, \quad x y z, \quad \& c .
$$

will represent the class of "navigable rivers that are estuaries." ...

As the combination of two literal symbols in the form $x y$ expresses the whole of that class of objects to which the names or qualities represented by $x$ and $y$ are together applicable, it follows that if the two symbols have exactly the same signification, their combination expresses no more than either of the symbols taken alone would do. In such case we should therefore have

$$
x y=x .
$$

As $y$ is, however, supposed to have the same meaning as $x$, we may replace it in the above equation by $x$, and we thus get

$$
x x=x .
$$

$\ldots[T]$ he above equation assumes the form

$$
x^{2}=x,
$$

We pass now to the consideration of another class of the signs of speech, and of the laws connected with their use.

\section{CLASS II.}

Signs of those mental operations whereby we collect parts into a whole, or separate a whole into its parts.

We ... are ... capable of ... forming the aggregate conception of a group of objects consisting of partial groups, each of which is separately named or described. For this purpose we use the conjunctions "and," "or," \&c. "Trees and minerals," "barren mountains, or fertile vales," are examples of this kind. In strictness, the words "and," "or," interposed between the terms descriptive of two or more classes of objects, imply that those classes are quite distinct, so that no member of one is found in another. In this and in all other respects the words "and" "or" are analogous with the sign + in algebra, and their laws identical. Thus the expression "men and women" is, conventional meanings set aside, equivalent with the expression "women and men." Let $x$ represent "men," $y$, "women;" and let + stand for "and" and "or," then we have

$$
x+y=y+x,
$$

an equation which would equally hold true if $x$ and $y$ represented numbers, and + were the sign of arithmetical addition. ...

[W] cannot conceive it possible to collect parts into a whole, and not conceive it also possible to separate a part from the whole. This operation we express in common language by the sign except, ... - As we have expressed the operation of aggregation by the sign + , so we may express the negative operation above described by - sign. Thus if $x$ be taken to represent men, and $y$, Asiatics, i.e. Asiatic men, then the concept of "All men except Asiatics" will be expressed by $x-y$. ...

We have seen that the symbols of Logic are subject to the special law,

$$
x^{2}=x \text {. }
$$

Now of the symbols of Number there are but two, viz. 0 and 1 , which are subject to the same formal law. We know that $0^{2}=0$, and that $1^{2}=1$; and the equation $x^{2}=x$, considered as algebraic, has no other roots than 0 and 1 . ... Let us conceive, then, of an Algebra in which the symbols $x, y, z, \& c$. admit indifferently of the values 0 and 1 , and of these values alone. ... 
$[T]$ he respective interpretations of the symbols 0 and 1 in the system of Logic are Nothing and Universe. ...

If $x$ represent any class of objects, then will $1-x$ represent the contrary or supplementary class of objects, i.e. the class including all objects which are not comprehended in the class $x$.

\section{0}

We first examine Boole's use of the symbols 0 and 1. On one hand, these symbols carry their numerical connotation from "Let us conceive, then, of an Algebra in which the symbols $x, y, z$ admit ... the values of 0 and 1." On the other hand, 0 and 1 have "interpretations ... in the system of Logic" as "Nothing" and "Universe." In this project, we will work with both versions, although for computations "literal symbols ... representing things" must have "the values 0 and 1, and of these values alone."

Exercise 4.1. If $x$ represents "mountains," $y$ "valleys," $z$ "things rich in minerals," and $w$ represents "things rich in vegetation," how would each of the following be represented using the symbols $x, y, z, w$, and the signs $\times,+,-$. Justify your answer in each case. Throughout consider "or" as inclusive, although in some examples there may be no object that satisfies both conditions of the "or" statement. Use parentheses where appropriate.

(a) mountains or valleys;

(b) mountains rich in minerals or valleys rich in minerals;

(c) mountains or valleys, provided that the formation is rich in minerals;

(d) mountains rich in vegetation, but not mountains rich in minerals;

(e) mountains, provided that they are rich in vegetation, but not in minerals;

(f) the class of objects that are not mountains. Hint: How does Boole represent "the contrary or supplementary class of objects"?

(g) the class of objects that are both rich in minerals and rich in vegetation;

(h) the class of objects that are not both rich in minerals and rich in vegetation;

(i) the class of objects that are either not rich in minerals or not rich in vegetation;

(j) the class of objects that are either rich in minerals or rich in vegetation, but not both.

(k) Boole states that "these symbols of Logic are in their use subject to definite laws, partly agreeing with ... the laws of the corresponding symbols in the science of Algebra." What law of symbolic logic can be concluded from parts (b) and (c) above? Justify your answer. From parts (d) and (e)? Justify your answer.

( $\ell$ ) What law of symbolic logic can be concluded from parts (h) and (i)? How does this compare to algebraic meaning of this "equation"? Recall that Boole also states that the symbols of logic partly differ from their algebraic use. 
Exercise 4.2. Letting $x$ and $y$ denote arbitrary "literal symbols" in the sense of Boole, we interpret $x=1$ as meaning that condition $x$ or property $x$ is "true," while $x=0$ means that condition $x$ is "false." We adopt Boole's notation that $x y$ denotes the condition that both $x$ and $y$ are "true". Let's compare the logical meaning of "and" with the algebraic law of multiplication. To separate logic from algebra for the moment, use either true, "T," or false, "F," when considering a statement in logic. First suppose that $x$ is either true or false, and $y$ is either true or false. Fill in the following table for truth of " $x$ and $y$," depending on the truth values of $x$ and $y$ individually.

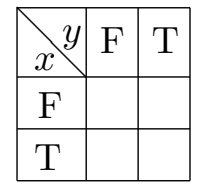

Truth of " $x$ and $y . "$

Now compare this to the standard (base 10) multiplication table for $x y$ when $x$ has the values 0 or 1 , and $y$ has the values of 0 or 1 .

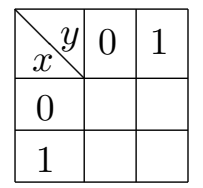

Multiplication Table for $x \cdot y$.

Do the logical values for " $x$ and $y$ " match the multiplication table for $x y$ with $\mathrm{T}=1$ and $\mathrm{F}=0$ ?

Using the "inclusive or," fill in the following table for the truth of " $x$ or $y$," depending on the individual truth values of $x$ and $y$.

\begin{tabular}{|c|c|c|}
\hline$x y$ & $\mathrm{~F}$ & $\mathrm{~T}$ \\
\hline $\mathrm{F}$ & & \\
\hline $\mathrm{T}$ & & \\
\hline
\end{tabular}

Truth of "inclusive or."

Now compare this to the standard (base 10) addition table for $x+y$, where $x$ has the values 0 or 1 , and $y$ has the values of 0 or 1 .

\begin{tabular}{|l|l|l|}
\hline$x y$ & 0 & 1 \\
\hline 0 & & \\
\hline 1 & & \\
\hline
\end{tabular}

Addition Table for $x+y$.

Do the logical values for " $x$ or $y$ " match the addition table for $x+y$ with $\mathrm{T}=1$ and $\mathrm{F}=0$ ? We will continue using $x+y$ to denote the "inclusive or," noting that if $x$ is true and $y$ is true, then $x+y$ is true. Keep in mind that Boole states "these symbols of Logic are in their use subject to definite laws, ... partly differing from the corresponding symbols in the science of Algebra."

Exercise 4.3. Recall that Chrysippus uses the negation of an "and" statement as one of his major premises. Letting $x$ and $y$ denote literal symbols in the sense of Boole, what construction in Boole's system represents the class of objects that do not satisfy both conditions $x$ and $y$ ? Fill in the following table for this new construction, using 1 s and 0 s. 


\begin{tabular}{|l|l|l|}
\hline$x y$ & 0 & 1 \\
\hline 0 & & \\
\hline 1 & & \\
\hline
\end{tabular}

Not both $x$ and $y$.

What construction in Boole's system represents the class of objects that either do not satisfy condition $x$ or do not satisfy condition $y$ ? Fill in the following table for this construction, using 1s and 0 s.

\begin{tabular}{|l|l|l|}
\hline$x y$ & 0 & 1 \\
\hline 0 & & \\
\hline 1 & & \\
\hline
\end{tabular}

Either not $x$ or not $y$.

Compare the tables for "not both $x$ and $y$ " with "either not $x$ or not $y$." Can the negation of every "and" statement be written as an "inclusive or" statement?

Exercise 4.4. The use of "+" to denote the "inclusive or" for the statement " $A$ or $B$ " leads to the rule of logic $1+1=1$. Interpret this in terms of the meaning of " $A$ or $B$ " when both $A$ and $B$ are true. What advantages does it afford to logic? What disadvantages? Apply your discussion to the rule

$$
1-x y=(1-x)+(1-y) .
$$

Comment on the usefulness of using "+" to denote "or," and "-" to denote negation.

\section{Frege Invents A Concept-Script}

An important contribution of George Boole is the reduction of certain statements in logic to a system of calculation using symbols in place of the words "and," "or," and "not," whereby complicated expressions can be manipulated algebraically. Boole borrowed symbols in common use from "the science of Algebra," namely $\times,+,-$, although in many cases the rules of algebra do not correspond to the rules of logic. Nonetheless, these symbols can then be used to express the major premise for the last three rules of inference stated by Chrysippus that involve the expressions "and," "or," "not." What about Chrysippus's first two rules of inference that involve a syllogism in the form of an "if-then" statement? Of Aristotelian logic itself, Boole questions "whether syllogism is indeed the fundamental type of reasoning" [6, p. 238], and concludes: "It cannot be conceived that syllogism should be the one essential process of reasoning, and yet the manifestation of that process present nothing indicative of this high quality of pre-eminence" [6, p. 239]. Boole does not introduce a special symbol for a syllogism, presumedly since the conclusions drawn therefrom can be achieved by other statements involving "and," "or," "not."

Let's now turn to the work of the German mathematician and philosopher Gottlob Frege (18481925) who sought a logical basis, not for language as Boole, but for arithmetic and mathematical analysis [11, p. 177]. Frege maintained that arithmetic propositions, such as $2+2=4$, should follow from expressions of truth in pure logic. Like the stoic philosophers of ancient Greece, Frege believed in an objective basis of thought. Unlike Boole, he used symbols showing no kinship with either arithmetic or algebra, and placed special emphasis on the concept of deduction.

Let's study more closely Frege's own particular notation, which he called a concept-script, or "Begriffsschrift" in the original German. Greek letters, such as $\xi, \zeta, \Delta, \Gamma$ represent propositions, 
such as statements in arithmetic, and belong to either the True or the False. A horizontal line - denotes a "judgment stroke" that renders the value of either true or false when applied to a proposition. For example, $-2^{2}=5$ returns the value "false," while $-2^{2}=4$ returns "true." The symbol $_{\mathrm{T}} \xi$ denotes the negation of $-\xi$, while $\zeta$ denotes that $\zeta$ is a true statement. These symbols may be combined in what Frege calls "amalgamation of horizontals," so that ${ }_{L_{T}}(\Delta)$ becomes $\uparrow_{T} \Delta$, meaning that the negation of $\Delta$ is true, i.e., $\Delta$ itself is false. Quoted below are only a few passages from The Basic Laws of Arithmetic [9], without mention of Frege's extensive work on quantified statements (today called "predicate logic.")

Exercise 5.1. (a) Evaluate the following symbols in Frege's concept script as either true or false. Briefly justify your answer.

(i) $-2+2=5$.

(ii) ${ }_{\mathrm{T}} 2+2=5$.

(b) Is ${ }_{+} 2+2=5$ a correct statement? Is $\vdash 2+2=4$ a correct statement? Justify your answers.

Frege does not introduce special symbols for either "and" or "or," but instead, in a highly original move, offers the world of logic an entirely new symbol, denoted

$$
\left[\begin{array}{l}
\xi \\
\zeta
\end{array}\right.
$$

called the condition-stroke $[8$, p. 20] [9, p. 51], which supersedes concepts like "and" and "or."

10000000000

\section{$\S 12$. Condition-stroke, And, Neither-nor, Subcomponents, Main Component.}

In order to enable us to designate the subordination of a concept under a concept, and other important relations, I introduce the function of two arguments

$$
T_{\zeta}^{\xi}
$$

by stipulating that its value shall be the False if the True be taken as $\zeta$-argument and any object other than the True be taken as $\xi$-argument, and that in all other cases, the value of the function shall be the True. ... The vertical stroke I call the condition-stroke. It may be lengthened as required.

The following propositions hold:

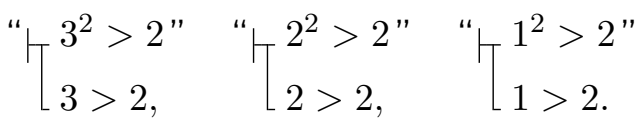

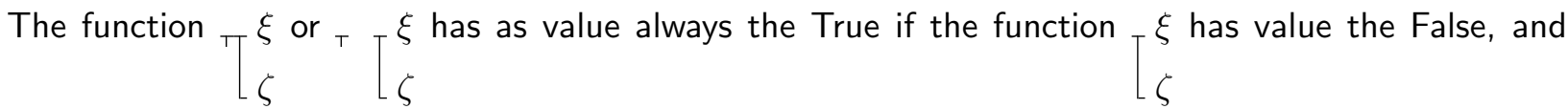
conversely. Therefore, $T_{\Delta}^{\Gamma}$ is the True if and only if $\Delta$ is the True and $\Gamma$ is not the True. Consequently

$$
\vdash_{\top} \begin{aligned}
& 2>3 \\
& 2+3=5
\end{aligned}
$$

In words: 2 is not greater than 3 and the sum of 2 and 3 is 5 . 
Exercise 5.2. To understand the condition-stroke, first review when $\xi$ is true. Of the four $\zeta$

possible combinations of $\xi$ and $\zeta$ being true or false, how many of them result in the truth of $\xi$ ?

Which ones? Given $\vdash_{\zeta} \xi$, i.e., that $\frac{\zeta}{\zeta}$ is true, and given $\vdash \zeta,(-\zeta$ is true), what can be concluded about $-\xi$ ? Why? Given $\vdash_{\Delta} \Gamma$ and ${ }_{\Gamma} \Delta$, i.e., $-\Delta$ is false, what can be concluded about $-\Gamma$ ? Justify your answer.

Now explain why the statement $\left\{\begin{array}{l}3^{2}>2 \text { is correct. What statement does } \xi \text { represent in this } \\ 3>2\end{array}\right.$ case? What is the value of $-\xi$ ? What does $\zeta$ represent here? What is the value of $-\zeta$ ? Explain

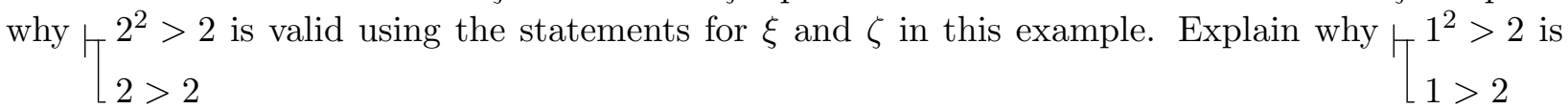
correct using the statements for $\xi$ and $\zeta$ in this case.

Exercise 5.3. Of the four possible combinations of $\xi$ and $\zeta$ being true or false, how many of them result in the truth of ${ }_{T} \xi$, i.e., the negation of $\xi$ ? Which ones? How does this compare to the $[\zeta \quad[\zeta$

truth values of the statement " $\xi$ and $\zeta$ "? Hint: Review Exercise 4.2 in the Boole section. Use the symbols $_{T}$ and ${ }_{T}$ in some combination to find a Frege statement that matches the truth values for " $\xi$ and $\zeta . "$ Justify your answer.

Exercise 5.4. Use the symbols ${ }_{T}$ and ${ }_{T}$ in some combination to find a construction that matches the truth values for " $\xi$ or $\zeta$ " for the "inclusive or." Comment on why Frege may not have introduced separate symbols for "and" and "or."

Let's read further from Frege [8, p. 25-26] [9, p. 57-59] and compare this to Chrysippus's indemonstrable argument forms.

1000000000

\section{$\S 14$. First Method of Inference.}

From the propositions " $\Gamma$ " and " $\angle \Delta$," we may infer " $\angle \Gamma$." For if $\Gamma$ were not the True, then since $\Delta$ is the True, $\Gamma$ would be the False. ...

$\S 15$. Second Method of Inference. Contraposition. 
We have that ${ }_{\Gamma} \Delta$ is the False if ${ }_{T} \Gamma$ is the True and ${ }_{T} \Delta$ is not the True; i.e., if $-\Gamma$ is the False and $\Delta[-\Delta]$ is the True. In all other cases ${ }_{T_{\Gamma}} \Delta$ is the True. But the same holds for ${ }_{\Gamma} \Gamma$; thus the functions $\zeta$ and $\xi$ always have the same value for the same arguments. ... Thus we may pass from $\xi \quad \zeta \zeta$

the proposition $\vdash^{\Gamma}$ to the proposition $\vdash \Delta$ and conversely. ...<smiles>C1CCC2(C1)CC2</smiles>

A subcomponent $[\zeta]$ may be interchanged with the main component $[\xi]$ if the truth-value of each is simultaneously reversed. This transition we call contraposition [Wendung].

1000000000

Exercise 5.5. Rewrite Chrysippus's first rule of inference "If the first, then the second; the first; therefore the second" entirely in Frege's notation. Identify two propositions $\xi, \zeta$ in the major premise "If the first, then the second," and find a symbol in Frege's concept-script that expresses how the first is related to the second. Be sure to justify your answer. Write the minor premise "the first [is true]" in Frege's notation. Write the conclusion "the second [is true]" in Frege's notation as well.

Exercise 5.6. Rewrite Chrysippus's second rule of inference "If the first, then the second; but not the second; therefore not the first" entirely in Frege's notation. Write the major premise, minor premise, and conclusion using his concept-script. Be sure to justify your answer, and carefully state the meaning of any symbols such as $\xi$ or $\zeta$ that you may wish to use. Rewrite "If the first, then the second" as an equivalent "if-then" statement beginning with "If not the second, ...." Write this latter "if-then" statement using the condition stroke. How does Chrysippus's second rule compare to Frege's law "A subcomponent may be interchanged with the main component if the truth-value of each is simultaneously reversed"?

Exercise 5.7. (a) Consider the example of Chrysippus's third rule of inference: "It is not the case both that it is day and it is night. It is day. Therefore, it is not night." Let $\xi$ denote the proposition "It is day," and let $\zeta$ denote the proposition "It is night." Write the major premise of the third rule entirely in Frege's notation. Write the minor premise and conclusion using his concept-script as well. Be sure to explain your solution using complete sentences.

(b) Although language is not as precise as symbolic logic, once a system of symbols is introduced, choices must sometimes be made in the interpretation of a passage when transcribing language into symbols. Comment on the above choice of $\xi$ and $\zeta$, namely "It is day" and "It is night" as independent symbols. Could the major premise above be written using just one of these symbols? Justify your answer.

Exercise 5.8. Using the "inclusive or," rewrite the example of Chrysippus's fifth rule of inference "Either it is day or it is night; but it is not night; therefore it is day" entirely in Frege's notation. Let $\xi$ denote "It is day," and let $\zeta$ denote "It is night," as independent symbols. Write the major premise, minor premise, and conclusion using his concept-script. Be sure to justify your answer using complete sentences.

Exercise 5.9. Compare and contract the following two statements. The first is attributed to Philo the Logician (Philo of Megara), and the second was authored by Gottlob Frege: 
I. A valid hypothetical proposition is "that which does not begin with a truth and end with a falsehood" [23, II. 110].

II. "I introduce the function of two arguments ${ }_{-} \xi$ by stipulating that its value shall be the False if $\zeta$

the True be taken as $\zeta$-argument and any object other than the True be taken as $\xi$-argument, and that in all other cases, the value of the function shall be the True" [9, p. 51].

Which of the arguments, $\xi$ or $\zeta$, in Frege's condition stroke plays the role of the "beginning" of a hypothetical proposition in Philo's statement? Which argument plays the role of the "ending" of a hypothetical proposition?

Exercise 5.10. Has Frege offered an alternative to Boole's statement "It cannot be conceived that syllogism [implication] should be the one essential process of reasoning, and yet the manifestation of that process present nothing indicative of this high quality of pre-eminence" [6, p. 239]? What is the manifestation of a syllogism [implication] in Frege's notation, at least in the form of an "if-then" statement?

\section{Russell and Whitehead Find New Notation}

While somewhat awkward in execution, Gottlob Frege's condition stroke ${ }^{4}$ is the centerpiece of his system of logic. The import of this symbol is that of deduction, and advances his philosophy that mathematical truths should follow from truths in logic, a point of view known today as logicism. Two later practitioners of logicism whose work set the stage for mathematical logic of the twentieth century were Bertrand Russell (1872-1970) and Alfred North Whitehead (1861-1947). The British mathematician and philosopher A. N. Whitehead began his career at Trinity College in Cambridge, where he taught from 1885 to 1910 [14]. In 1898 he authored A Treatise on Universal Algebra, with Applications [27] concerning the foundations of mathematics. Bertrand Russell matriculated at Trinity as a student in 1890, where Whitehead became his mentor. Russell authored The Principles of Mathematics [20] in 1903, which also dealt with foundational issues, and soon the two scholars began a collaboration on what would become their highly influential magnum opus Principia Mathematica [21], published in three volumes (1910, 1912, 1913). The work develops the doctrine of logicism, and seeks to place on firm foundations emerging ideas in set theory. While students new to mathematics see little controversy in the basic principles of the subject, Russell had discovered an inherent paradox in the work of Gottlob Frege and Georg Cantor (1845-1918) on the fundamental notion of what a set ought to be. Russell was fond of the following non-technical description of the paradox: Suppose that the barber of a certain town is a man who (lives in that town) and shaves all men in town who do not shave themselves. Does the barber shave himself? If the answer is "yes," then the barber does not shave himself, so the answer is "no." If the answer is "no," then it is false that the barber does not shave himself, so the answer is "yes" the barber does shave himself. Although this project will not develop Cantor's ideas, nor Russell's purported solution to this paradox, the project does offer a brief excerpt from the introduction of Principia Mathematica, where the authors state that mathematical logic "is framed with a view to the perfectly precise expression, in its symbols, of mathematical propositions: to secure such expression, and to secure it in the simplest and most convenient notation possible ..." [25, p. 
1]. While reading the work of Russell and Whitehead, compare their notation to that of their predecessors Frege and Boole.

Russell was a prolific writer, contributing to the fields of education, history, religion, and political theory, not to mention philosophy and logic [15]. In 1950 he received the Nobel Prize in Literature "in recognition of his varied and significant writings in which he champions humanitarian ideals and freedom of thought," to quote the Nobel Foundation. He was an advocate of social reform, an anti-war activist, and later in life an anti-nuclear activist. His anti-war protests in England resulted in his dismissal from Trinity College in 1916, and a five-month imprisonment in 1918. Furthermore, in response to public protest, his appointment at the City College of New York was terminated in 1940. Let's read from the monumental Principia Mathematica [25, p. 6-8]:

\section{0}

The fundamental functions of propositions. ...

[T] here are four special cases which are of fundamental importance, since all the aggregations of subordinate propositions into one complex proposition which occur in the sequel are formed out of them step by step.

They are (1) The Contradictory Function, (2) the Logical Sum or Disjunctive Function, (3) the Logical Product, or Conjunctive Function, (4) the Implicative Function. ...

The Contradictory Function with argument $p$, where $p$ is any proposition, is the proposition which is the contradictory of $p$, that is, the proposition asserting that $p$ is not true. This is denoted by $\sim p$. Thus, $\sim p \ldots$ means the negation of the proposition $p$. It will also be referred to as the proposition not $p$. ...

The Logical Sum is a proposition with two arguments $p$ and $q$, and is the proposition asserting $p$ or $q$ disjunctively, that is, asserting that at least one of the two $p$ and $q$ is true. This is denoted $p \vee q \ldots$ Accordingly $p \vee q$ means that at least $p$ or $q$ is true, not excluding the case in which both are true.

The Logical Product is a propositional function with two arguments $p$ and $q$, and is the proposition asserting $p$ and $q$ conjunctively, that is, asserting that both $p$ and $q$ are true. This is denoted by $p . q \ldots$ Accordingly $p . q$ means that both $p$ and $q$ are true. ...

The Implicative Function is a propositional function with two arguments $p$ and $q$, and is the proposition that either not- $p$ or $q$ is true, that is, it is the proposition $\sim p \vee q$. Thus, if $p$ is true, $\sim p$ is false, and accordingly the only alternative left by the proposition $\sim p \vee q$ is that $q$ is true. In other words if $p$ and $\sim p \vee q$ are both true, then $q$ is true. In this sense the proposition $\sim p \vee q$ will be quoted as stating that $p$ implies $q$. The idea contained in this propositional function is so important that it requires a symbolism which with direct simplicity represents the proposition ... . The symbol employed for " $p$ implies $q$ ", i.e. for " $\sim p \vee q$ " is " $p \supset q$." This symbol may also be read "if $p$, then $q . " \ldots$

Truth-values. The "truth-value" of a proposition is truth if it is true, and falsehood if it is false $\mathrm{e}^{5}$. It will be observed that the truth-values of $p \vee q, p . q, p \supset q, \sim p, \ldots$ depend only upon those of $p$ and $q$

\section{0}

In part I of Principia Mathematica, the theme of deduction is studied in detail with this notion expressed collectively as "the principles by which conclusions are inferred from premisses" [25, p. 90]. A crucial notion in this study is the implicative function stated above. Later in part I, Russell and Whitehead write [25, p. 94]:

\section{0}

${ }^{5}$ This phrase is due to Frege. (Footnote actually in Principia Mathematica). 
Definition of Implication. When a proposition $q$ follows from a proposition $p$, so that if $p$ is true, $q$ must also be true, we say that $p$ implies $q$. The idea of implication, in the form in which we require it, can be defined. ... The essential property that we require of implication is this: "What is implied by a true proposition is true." It is in virtue of this property that implication yields proofs. But this property by no means determines whether anything, and if so what, is implied by a false proposition. What it does determine is that if $p$ implies $q$, then it cannot be the case that $p$ is true and $q$ is false, ...

\section{coroco0000000}

Exercise 6.1. In the notation of Principia Mathematica, the symbols $p, q, r, s$, etc. denote "elementary propositions," which we shall interpret as simple statements that are either true of false. For this exercise, let $p, q, r$, and $s$ denote the following.

$p$ : "The geological formation is a mountain."

q: "The geological formation is a valley."

$r$ : "The geological formation is rich in minerals."

$s$ : "The geological formation is rich in vegetation."

Write each of the following in the notation of Principia Mathematica, using $\sim, \cdot, \vee$. Be sure to justify your answer. Consider "or" as being inclusive.

(a) The geological formation is either a mountain or a valley.

(b) The geological formation is either a mountain rich in minerals or a valley rich in minerals.

(c) The geological formation is either a mountain or a valley, provided that it is rich in minerals.

(d) The geological formation is a mountain rich in vegetation, but not a mountain rich in minerals.

(e) The geological formation is a mountain, provided that it is rich in vegetation but not in minerals.

(f) The geological formation is not a mountain.

(g) The geological formation is rich in minerals and rich in vegetation.

(h) The geological formation is not both rich in minerals and rich in vegetation.

(i) The geological formation is either not rich in minerals or not rich in vegetation.

(j) The geological formation is either rich in minerals or rich in vegetation, but not both.

(k) What law of symbolic logic can be concluded from parts (b) and (c) above? Justify your answer. What law of symbolic logic can be concluded from parts (h) and (i)? Justify your answer. Compare Russell and Whitehead's notation with that of Boole.

( $\ell$ ) What law of symbolic logic can be concluded from parts (d) and (e)? Justify your answer. Compare Russell and Whitehead's notation to that of Boole.

(m) Do you feel that Russell and Whitehead have found the logic for "perfectly precise expression, in its symbols, of mathematical propositions: to secure such expression, and to secure it in the simplest and most convenient notation possible"? Explain. 
Exercise 6.2. Discuss the intellectual progress towards an understanding of the truth of an implication from the following quotes of Philo, Frege, and Russell-Whitehead:

I. A valid hypothetical proposition is "that which does not begin with a truth and end with a falsehood" [23, II. 110].

II. "I introduce the function of two arguments $\xi$ by stipulating that its value shall be the False if $\zeta$

the True be taken as $\zeta$-argument and any object other than the True be taken as $\xi$-argument, and that in all other cases, the value of the function shall be the True" $[9$, p. 51].

III. "[I]f $p$ implies $q$, then it cannot be the case that $p$ is true and $q$ is false ..." [25, p. 94].

How would $\xi$ be written in the notation of Principia Mathematica? Be sure to identify the symbol

from Principia Mathematica that plays the role of the condition-stroke. Justify your answer in terms of the truth-values of the respective symbols from the works of Frege and Russell-Whitehead. What would a "hypothetical proposition" be called in the language of Principia Mathematica?

Exercise 6.3. Write the major premise from Chrysippus's first rule of inference "If the first, then the second" entirely in the notation of Principia Mathematica. Be sure to identify two elementary propositions $p$ and $q$ in your response and state their meaning.

Exercise 6.4. Reread the example of Chrysippus's second rule of inference. Write "If it is day, then it is light" entirely in the notation of Principia Mathematica. Be sure to identify two elementary propositions $p$ and $q$ in this case, and state their meaning. Now, using the same choice of $p$ and $q$, write "If it is not light, then it is not day" entirely in the notation of Principia Mathematica. Compare this to Frege's approach outlined in Exercise 5.6.

Exercise 6.5. Reread the example of Chrysippus's fifth rule and compare this to the implicative function of Russell and Whitehead. Using the "inclusive or," rewrite "Either it is day or it is night" in the notation of Principia Mathematica. State the meaning of any elementary propositions in your response. Using the same propositions, write "It is not night" in the notation of Principia Mathematica. Write "Either it is day or it is night" as an equivalent "if-then" statement so that the minor premise "It is not night" results in the same conclusion as Chrysippus's fifth rule of inference. Can every "inclusive or" statement be written as an "if-then" statement? Justify your answer. Can every "if-then" statement be written as an equivalent "inclusive or" statement? Justify your answer.

Exercise 6.6. Rewrite the major premise from the example of Chrysippus's third rule: "It is not the case both that is is day and it is night" in the notation of Principia Mathematica. Begin with two elementary propositions denoting "It is day" and "It is night." Rewrite the major premise as an "inclusive or" statement using a rule of logic discovered in Exercise 6.1. Find an implication ("ifthen" statement) that is logically equivalent to the major premise. Can the negation of every "and" statement be written as an implication? Justify your answer. Conversely, can every implication be written as the negation of an "and" statement? Justify your answer in this case.

Exercise 6.7. In the fourth rule of inference, Chrysippus states "Either the first or the second; but the first; therefore not the second," which employs the "exclusive or." Write the "exclusive or" in terms of the Russell-Whitehead symbols $\sim, \cdot, \vee$. Be sure to explain your answer. 


\section{Wittgenstein Constructs Schemata}

In Principia Mathematica Russell and Whitehead state that the truth-values of the fundamental functions of propositions, i.e., " $p$ and $q$," " $p$ or $q$," " $p$ implies $q$," and "not $q$," depend only upon those of the elementary propositions $p$ and $q$. Exactly how is the truth value of a compound statement determined from its more elementary pieces? The algebraic notation of Boole, involving $\times,+,-$, does not entirely agree with the logical meaning of "and," "or," "not." The condition stroke of Frege, although brilliant in its encapsulated meaning, is difficult to manipulate. How should the rules for calculation in symbolic logic be expressed?

Let's now turn to the work of Ludwig Wittgenstein (1889-1951), considered by some as the greatest philosopher of the twentieth century [4]. The Austrian-born scholar contributed to the fields of logic, philosophy, and the study of language, while at times he worked modestly as a school teacher, gardener, and architect, not to mention his service in the Austro-Hungarian army during World War I. Upon the advise of Frege, the young Wittgenstein began to study logic with Russell at Trinity College in 1911. While Russell initially wrote of Wittgenstein as "an unknown German ... obstinate ... but I think not stupid," the British scholar soon came to think very highly of Wittgenstein, particularly in the realm of logicism [4].

Wittgenstein's first publication Logische-philosophische Abhandlung [28], translated as Tractatus Logico-Philosophicus [29] [30], introduced a simple and unifying principle for evaluating the truthpossibilities of symbolic statements in logic. As with his predecessors, he resolves complicated statements into elementary propositions that are either true or false. For example, "the geological formation is a mountain" could represent an elementary proposition. These propositions are then combined into "states of affairs" (Sachverhalten in the original German) using connectives like "and," "or," and "not." Wittgenstein speaks of symbolic logic as a "picture of reality," much like musical notation is a "picture" of music. Moreover, he introduces schemata (tables ${ }^{6}$ ) for displaying all truth-possibilities of these pictures, allowing an easy overview of the conditions under which a "picture" would be true and when it would be false. The Tractatus was highly influential, and became a topic of discussion in the Vienna Circle of scholars. The text is organized around seven main statements, numbered consecutively. Sub-numbering is then used to modify these statements in turn. For example, statement 1.1 modifies statement 1 , while 1.11 modifies 1.1 , etc. The Tractatus begins with the broad and ambitious first statement "1. The world is all that is the case," and quickly limits its scope to propositions that "restrict reality to two alternatives: yes and no."

Initial publication of Logische-philosophische Abhandlung was met with difficulty and included rejections from Cambridge University Press as well as several German publishing houses [11, p. 436]. With the assistance of Bertrand Russell, however, the manuscript first appeared in the German periodical Annalen der Naturphilosophie (Annals of Natural Philosophy) in 1921. Let's read a few excerpts from a modern translation of the Tractatus [30].

0000000000

4 A thought is a proposition with a sense.

4.2 The sense of a proposition is its agreement and disagreement with possibilities of existence and non-existence of states of affairs.

\footnotetext{
${ }^{6}$ The influential logician Charles S. Peirce (1839-1922) also developed a type of truth table for his three-valued system of logic, based on statements having values $0,1 / 2$, and 1 .
} 
$4.002 \ldots$ Everyday language is a part of the human organism and is no less complicated than it.

It is not humanly possible to gather immediately from it what the logic of language is.

4.003 Most of the propositions and questions to be found in philosophical works are not false but nonsensical. Consequently we cannot give any answer to questions of this kind, but can only establish that they are nonsensical.

4.01 A proposition is a picture of reality. ...

4.011 At first sight a proposition —one set out on the printed page, for example-does not seem to be a picture of the reality with which it is concerned. But no more does musical notation at first sight seem to be a picture of music, nor our phonetic notation (the alphabet) to be a picture of our speech.

And yet these sign-languages prove to be pictures, even in the ordinary sense, of what they represent.

4.023 A proposition must restrict reality to two alternatives: yes or no.

4.024 To understand a proposition means to know what is the case if it is true.

4.06 A proposition can be true or false only in virtue of being a picture of reality.

4.11 The totality of true propositions is the whole of natural science (or the whole corpus of the natural sciences).

4.121 ... Propositions show the logical form of reality. They display it.

4.21 The simplest kind of proposition, an elementary proposition, asserts the existence of a state of affairs.

$4.24 \ldots$ I indicate them [elementary propositions] by the letters $p, q, r$.

4.25 If an elementary proposition is true, the state of affairs exists: If an elementary proposition is false, the state of affairs does not exist.

4.26 ... The world is completely described by giving all elementary propositions, and adding which of them are true and which are false.

4.31 We can represent truth-possibilities by schemata of the following kind ("T" means "true," "F" means "false;" the rows of T's and F's under the row of elementary propositions symbolize their truth-possibilities in a way that can easily be understood):

\begin{tabular}{c|c|c}
$p$ & $q$ & $r$ \\
\hline $\mathrm{T}$ & $\mathrm{T}$ & $\mathrm{T}$ \\
\hline $\mathrm{F}$ & $\mathrm{T}$ & $\mathrm{T}$ \\
\hline $\mathrm{T}$ & $\mathrm{F}$ & $\mathrm{T}$ \\
\hline $\mathrm{T}$ & $\mathrm{T}$ & $\mathrm{F}$ \\
\hline $\mathrm{F}$ & $\mathrm{F}$ & $\mathrm{T}$ \\
\hline $\mathrm{F}$ & $\mathrm{T}$ & $\mathrm{F}$ \\
\hline $\mathrm{T}$ & $\mathrm{F}$ & $\mathrm{F}$ \\
\hline $\mathrm{F}$ & $\mathrm{F}$ & $\mathrm{F}$ \\
\hline
\end{tabular}

\begin{tabular}{c|c}
$p$ & $q$ \\
\hline \hline $\mathrm{T}$ & $\mathrm{T}$ \\
\hline $\mathrm{F}$ & $\mathrm{T}$ \\
\hline $\mathrm{T}$ & $\mathrm{F}$ \\
\hline $\mathrm{F}$ & $\mathrm{F}$ \\
\hline
\end{tabular}

$$
\frac{p}{\frac{\mathrm{T}}{\mathrm{F}}} .
$$


4.442 For example, the following is a propositional $\operatorname{sign}^{7}$ :

\begin{tabular}{c|c||c}
$p$ & $q$ & \\
\hline \hline $\mathrm{T}$ & $\mathrm{T}$ & $\mathrm{T}$ \\
\hline $\mathrm{F}$ & $\mathrm{T}$ & $\mathrm{T}$ \\
\hline $\mathrm{T}$ & $\mathrm{F}$ & \\
\hline $\mathrm{F}$ & $\mathrm{F}$ & $\mathrm{T}$ \\
\hline
\end{tabular}

If the order of the truth-possibilities in a schema is fixed once and for all by a combinatory rule, then the last column by itself will be an expression of the truth-conditions. If we now write this column as a row, the propositional sign will become

$$
\text { "(TT-T) }(p, q) "
$$

or more explicitly

$$
\text { “(TTFT) }(p, q) . "
$$

4.51 Suppose that I am given all elementary propositions: then I can simply ask what propositions I can construct out of them. And there I have all propositions.

5 A proposition is a truth-function of elementary propositions.

5.101 The truth-functions of a given number of elementary propositions can always be set out in a schema of the following kind:

$$
\begin{aligned}
& (T T T F)(p, q) \text { In words: } p \text { or } q .(p \vee q) \\
& (F F T T)(p, q) \quad " \quad \text { "Not } q .(\sim q) \\
& (\operatorname{FTFT})(p, q) \quad " \quad \text { Not } p .(\sim p) \\
& (T F F F)(p, q) \quad " \quad " \quad q \text { and } p \cdot(q \cdot p)
\end{aligned}
$$

I will give the name truth-grounds of a proposition to those truth-possibilities of its truth-arguments that make it true.

5.12 In particular, the truth of a proposition " $p$ " follows from the truth of another proposition " $q$ " if the truth-grounds of the latter $[q]$ are [among] the truth-grounds of the former $[p]$.

5.121 The truth-grounds of the one are contained in those of the other: $p$ follows from $q$.

5.122 If $p$ follows from $q$, the sense of " $p$ " is contained in the sense of " $q$. ."

\section{0}

\footnotetext{
${ }^{7}$ The entry in the third row and third column is left blank, although filled in later to be "false."
} 
Exercise 7.1. Wittgenstein's simple yet profound contribution to symbolic logic is the use of schemata (tables) to easily display all the truth-possibilities of a statement or proposition. Letting $p$ and $q$ denote elementary propositions, fill in the last column for " $p$ or $q$ " and " $p$ and $q$ " in each of the following tables. Use the "inclusive or."

\begin{tabular}{c|c||l}
$p$ & $q$ & $p \vee q$ \\
\hline \hline $\mathrm{T}$ & $\mathrm{T}$ & \\
\hline $\mathrm{F}$ & $\mathrm{T}$ & \\
\hline $\mathrm{T}$ & $\mathrm{F}$ & \\
\hline $\mathrm{F}$ & $\mathrm{F}$ & \\
\hline
\end{tabular}

\begin{tabular}{r|r||l}
$p$ & $q$ & $p . q$ \\
\hline \hline $\mathrm{T}$ & $\mathrm{T}$ & \\
\hline $\mathrm{F}$ & $\mathrm{T}$ & \\
\hline $\mathrm{T}$ & $\mathrm{F}$ & \\
\hline $\mathrm{F}$ & $\mathrm{F}$ & \\
\hline
\end{tabular}

Explain your solution and compare your work to statement 5.101 in the Tractatus.

Exercise 7.2. Wittgenstein introduces the term truth-grounds of a proposition to indicate the "truth-possibilities of its truth-arguments that make it true." What are the truth-grounds of $p \vee q$ ? of $q . p$ ? Justify your answer.

Exercise 7.3. Let $p$ and $q$ be elementary propositions and consider the compound proposition " $p$ follows from $q$ " ( $q$ implies $p$ ). What might the schema (table) for this compound statement be based on Wittgenstein's observation that $p$ follows from $q$ when the truth-grounds of $q$ (the values for which $q$ is true) are contained in the truth-grounds of $p$ (the values for which $p$ is true). Use this to fill in the following table:

\begin{tabular}{r|r||l}
$p$ & $q$ & $p$ follows from $q$ \\
\hline \hline $\mathrm{T}$ & $\mathrm{T}$ & \\
\hline $\mathrm{F}$ & $\mathrm{T}$ & \\
\hline $\mathrm{T}$ & $\mathrm{F}$ & \\
\hline $\mathrm{F}$ & $\mathrm{F}$ & \\
\hline
\end{tabular}

Do you feel that Wittgenstein has clearly stated how the truth values of " $p$ follows from $q$ " are determined from the truth values of $p$ and $q$ separately? What passages from Principia Mathematica might help in the determination of the truth values of $q \supset p$ ?

Exercise 7.4. For elementary proposition $p, q$, and $r$, determine the truth-grounds of the compound propositions below. To organize your work, you may wish to first construct the Wittgenstein schemata for these statements. Consider "or" as inclusive.

(a) $\sim(p . q)$

(b) $(p . r) \vee(q . r)$

(c) $(\sim p) \vee(\sim q)$

(d) $(p \vee q) \cdot r$

Which of the above compound propositions are logically equivalent? Compare your answer to the laws of symbolic logic discovered in Exercise 6.1.

Exercise 7.5. Discover your own law of symbolic logic not discussed in Exercise 7.4. You may wish to study Exercise 6.1 for further laws not in Exercise 7.4, or simply find a new law. Be sure to justify your claims. 


\title{
8 Post Develops Truth Tables
}

Independently of Ludwig Wittgenstein, Emil Post (1897-1954) developed a highly efficient method to represent the truth values of compound statements involving the connectives "and," "or," "not," and "if-then." He dubbed these schematic representations "truth tables," a term which is in current use today.

Emil was born in Poland on February 11, 1897, and emigrated with his parents to New York in 1904. He received his undergraduate degree from the City College of New York, where he would later return as a faculty member. While a graduate student at Columbia University, Post became keenly familiar with the work of Russell and Whitehead, and participated in a seminar devoted to the study of Principia Mathematica [19, p. xi-xii]. The notion of a "truth table" along with a clear rendition of the table for " $p$ implies $q$ " appear in his doctoral dissertation "Introduction to a General Theory of Elementary Propositions" [18], published in 1921, the same year as Wittgenstein's "Logische-philosophische Abhandlung." Post begins his thesis with the "elementary propositions" of Russell and Whitehead, namely the atomic propositions that are either true of false. He quickly develops truth tables, and address several other issues, including multi-valued logic and a standard or canonical form for statements in mathematical logic [19, p. xiii]. The former had also been studied by Charles Peirce (1839-1922), while standard forms in logic are quite useful in computer science. Additionally, he contributed to the fields of analysis (calculus) and abstract algebra, while his work in logic anticipated several pioneering results that would limit the scope of Principia Mathematica, which was initially written to reduce all of mathematics to logic.

Sadly, Emil suffered from manic depression, a condition which during his life time was treated by electro-convulsive shock. His death from a sudden heart attack occurred in a mental institution shortly after one of these treatments [19, p. xii]. Let's read a brief excerpt from Post's thesis [18], and notice his rendering of the truth table for " $p$ implies $q . "$ Here and below $p$ and $q$ are elementary propositions, assuming the value of "true" or "false."

1000000000

\section{INTRODUCTION TO A GENERAL THEORY OF ELEMENTARY PROPOSITIONS.}

\author{
By EmIL L. Post.
}

\section{INTRODUCTION.}

In the general theory of logic built up by Whitehead and Russell [21] to furnish a basis for all mathematics there is a certain subtheory ... this subtheory uses ... but one kind of entity which the authors have chosen to call elementary propositions. ...

2. Truth-Table Development-Let us denote the truth-value of any proposition $p$ by + if it is true and by - if it is false. This meaning of + and - is convenient to bear in mind as a guide to thought, ... Then if we attach these two primitive truth-tables to $\sim$ and $\vee$ we have a means of calculating the truth-values of $\sim p$ and $p \vee q$ from those of their arguments.

\begin{tabular}{c|c}
$p$ & $\sim p$ \\
\hline+ & - \\
- & +
\end{tabular}

\begin{tabular}{c|c}
$p, q$ & $p \vee q$ \\
\hline++ & + \\
+- & + \\
-+ & + \\
-- & -
\end{tabular}


... It will simplify the exposition to introduce...

$$
p \supset q .=. \sim p \vee q
$$

read " $p$ implies $q, "$... having the table

$$
\begin{array}{c|c}
p, q & p \supset q \\
\hline++ & + \\
+- & - \\
-+ & + \\
-- & +
\end{array}
$$

1000000000

Exercise 8.1. Letting $p$ and $q$ denote elementary propositions, use the notation of Emil Post to fill in the right-hand column of each of the following truth tables: Consider "or" as inclusive.

\begin{tabular}{l|l}
$p, q$ & $p \cdot q$ \\
\hline++ & \\
+- & \\
-+ & \\
-- &
\end{tabular}

\begin{tabular}{l|l}
$p, q$ & $\sim(p . q)$ \\
\hline++ & \\
+- & \\
-+ & \\
-- &
\end{tabular}

\begin{tabular}{c|c|c|c}
$p, q$ & $\sim p$ & $\sim q$ & $(\sim p) \vee(\sim q)$ \\
\hline++ & & & \\
+- & & & \\
-+ & & & \\
-- & & &
\end{tabular}

Which compound propositions above are logically equivalent? Why? Compare your answer with Exercise 7.4.

Exercise 8.2. Let $p$ and $q$ denote elementary propositions. From the work of Russell and Whitehead, explain why $p \supset q$ and $\sim p \vee q$ are logically equivalent, which Post uses above. Construct the truth table for $\sim p \vee q$ and compare this to the table for $p \supset q$ given by Post.

Exercise 8.3. Construct the Post truth table for $q \supset p$ for elementary propositions $p$ and $q$. Compare this table to Wittgenstein's description that " $p$ follows from $q$," i.e. $q \supset p$, when the truth-grounds of $q$ are contained in the truth-grounds of $p$. You may wish to re-read Exercise 7.3.

Exercise 8.4. (a) Write "If it is day, then it is light" entirely in the notation of Principia Mathematica. Be sure to identify two elementary propositions $p$ and $q$, and state their meaning.

(b) Now, using the same choice of $p$ and $q$, write "If it is not light, then it is not day" entirely in the notation of Principia Mathematica.

(c) Using the notation of Emil Post, construct the truth tables for the symbolic statements in (a) and (b), and compare their final columns. Based on the truth values in these tables, what can be concluded about Chrysippus's first two rules of inference? Justify your answer. How had Frege described the relation between these two rules of inference?

Exercise 8.5. Letting $p$ denote the elementary proposition "It is day," and $q$ denote "It is night," write the compound statement "It is not the case both that it is day and it is night" in the notation of Principia Mathematica. Construct the schema or truth table for this compound statement as well. Find an implication ("if-then" statement) involving $p$ and $q$ that has an identical schema or truth table, and justify your answer. Hint: Reread Chrysippus's third rule of inference. 
Exercise 8.6. Can an "inclusive or" statement always be rewritten as an equivalent "if-then" statement? Justify your answer using appropriate schemata or truth tables for these two types of statements. Hint: Reread Chrysippus's fifth rule of inference.

Exercise 8.7. Can an "exclusive or" statement always be rewritten as an equivalent "if-then" statement? Justify your answer using appropriate schemata or truth tables for these two types of statements.

Exercise 8.8. Rewrite the implication "If the roof leaks, then the house will not sell" as an equivalent

(a) "inclusive or" statement;

(b) negation of an "and" statement;

(c) "if-then" statement beginning with "If the house will sell, ...."

Justify your answer in each case.

Exercise 8.9. Rewrite the implication "If a person is born at the rising of the Dog Star, then that person will not die at sea" as an equivalent

(a) "inclusive or" statement;

(b) negation of an "and" statement;

(c) "if-then" statement beginning with "If a person will die at sea, ...."

Justify your answer in each case.

Exercise 8.10. Returning to Aristotle's work on logic, consider the statements

(I) If $C$ occurs, then $B$ occurs; and if $B$ occurs, then $A$ occurs.

(II) If $C$ occurs, then $A$ occurs.

Is statement (I) logically equivalent to statement (II)? Identify elementary propositions for each and support your answer with appropriate schemata or truth tables.

Exercise 8.11. Is the concept of an "if-then" statement inherent in the use of language? Did logic evolve to gain insight into the use of language? Cite relevant passages from the works of Aristotle, Chrysippus, Boole, Frege, Russell-Whitehead, Wittgenstein or Post to support your claims.

Exercise 8.12. Is the concept of an "if-then" statement inherent in logic? Did the use of "ifthen" statements evolve to foster an understanding of logic? Cite relevant passages from the works of Aristotle, Chrysippus, Boole, Frege, Russell-Whitehead, Wittgenstein or Post to support your claims.

\section{Notes to the Instructor}

This project is designed for an introductory course in discrete or finite mathematics that covers elementary symbolic logic. Particular emphasis is placed on the logic of deduction as expressed today in an "if-then" statement. An examination of various rules of inference throughout history reveals a commonality of deductive thought, encapsulated today in the schemata of Ludwig Wittgenstein and truth tables of Emil Post. The instructor may wish to lead class discussion on certain excerpts from the original sources, and assign certain problems as out-of-class exercises. Be sure to draw 
comparisons from one historical source to another, and follow the progress to what today appears rather abruptly in most textbooks on discrete mathematics, namely the truth-table for an "if-then" statement.

Although there are numerous exercises, not all need to be assigned. Furthermore, as an in-class activity, the students could work through several exercises together with the instructor. Other exercises could be added at the conclusion of the project, such as comparing the truth tables or Frege's concept-script for:

1. $p \supset(q \supset r)$ and $(p \supset q) \supset r$;

2. $p \supset(q \supset r)$ and $(p . q) \supset r$.

Above $p, q, r$ are elementary propositions, and using the notation of Principia Mathematica [25], $p . q$ denotes " $p$ and $q, " p \vee q$ denotes " $p$ or $q$," and $p \supset q$ denotes " $p$ implies $q . "$ Many modern textbooks write $p . q$ as $p \wedge q$ and $p \supset q$ as $p \rightarrow q$. The main goal of the project, however, is to gain an appreciation of symbolic logic by witnessing the various attempts and historical progress at reducing statements to a useful symbolic form. For further material on Boolean algebras, see the projects of Janet Barnett, and for more information about coding information with 0s and 1s for computer science, see Inna Pivkina's project "Discovery of Huffman Codes."

\section{References}

[1] Aristotle, The Basic Works of Aristotle, McKeon, R. (editor), Random House, New York, 1941.

[2] Aristotle, On Interpretation, in [1].

[3] Aristotle, Prior Analytics, in [1].

[4] Biletzki, A., Matar, A., "Ludwig Wittgenstein," Stanford Encyclopedia of Philosophy, http:// plato.stanford.edu.

[5] Boole, G., An Investigation of the Laws of Thought, Walton and Maberly, London, 1854.

[6] Boole, G., An Investigation of the Laws of Thought, Dover Publications, Inc., New York, 1958.

[7] Diogenes Laertius, Lives of Eminent Philosophers, Hicks, R. D. (translator), Harvard University Press, Cambridge, Massachusetts, 1965.

[8] Frege, G., Grundgesetze der Arithmetik: Begriffschriftlich abgeleitet, (photographic reproduction) Georg Olms, Hildesheim, 1962.

[9] Frege, G., The Basic Laws of Arithmetic: Exposition of the System, Furth, M. (translator), University of California Press, Los Angeles, 1964.

[10] Gillispie, C.C., Holmes, F.L., (editors) Dictionary of Scientific Biography, Charles Scribner's Sons, New York, 1970.

[11] Grattan-Guinness, I., The Search for Mathematical Roots, 1870-1940, Princeton University Press, Princeton, New Jersey, 2000.

[12] Gould, J. B., "Chrysippus: On the Criteria for the Truth of a Conditional Proposition," Phronesis, 12 (1967), 152-161. 
[13] Gould, J. B., The Philosophy of Chrysippus, State University of New York Press, Albany, New York, 1970.

[14] Irvine, A. D., "Alfred North Whitehead," Stanford Encyclopedia of Philosophy, http:// plato.stanford.edu.

[15] Irvine, A. D., "Bertrand Russell," Stanford Encyclopedia of Philosophy, http:// plato.stanford.edu.

[16] Katz, V., A History of Mathematics: An Introduction, second ed., Addison Wesley, New York, 1998.

[17] Long, A. A., Sedley, D. N., The Hellenistic Philosophers, Vol. I, Cambridge University Press, Cambridge, England, 1987.

[18] Post, E. L., "Introduction to a General Theory of Elementary Propositions," American Journal of Mathematics, 43 (1921), 163-185.

[19] Post, E. L., Solvability, Provability, Definability: The Collected Works of Emil L. Post, Davis, M. (editor), Birkhäuser, Boston, 1994.

[20] Russell, B., The Principles of Mathematics, Cambridge University Press, Cambridge, England, 1903.

[21] Russell, B., Whitehead, A.N., Principia Mathematica, Cambridge University Press, Cambridge, England, 1910 (Vol. 1), 1912 (Vol. 2), 1913 (Vol. 3).

[22] Russell, B., A Critical Exposition of the Philosophy of Leibniz, second ed., Allen and Unwin, London, 1937.

[23] Sextus Empiricus, Outlines of Pyrrhonism Bury, R. G. (translator), Harvard University Press, Cambridge Massachusetts, 1967.

[24] Sextus Empiricus, Against the Logicians, Bury, R. G. (translator), Harvard University Press, Cambridge Massachusetts, 1967.

[25] Russell, B., Whitehead, A. N., Principia Mathematica to *56, Cambridge University Press, Cambridge, England, 1997.

[26] Smith, R., "Aristotle's Logic," Stanford Encyclopedia of Philosophy, http:// plato.stanford.edu.

[27] Whitehead, A.N. A Treatise on Universal Algebra, with Applications, Cambridge University Press, Cambridge, England, 1898.

[28] Wittgenstein, L., "Logische-philosophische Abhandlung," Annalen der Naturphilosophie, 14 (1921), 198-262.

[29] Wittgenstein, L., Tractatus Logico-Philosophicus, Ramsey, F., Ogden, C.K. (translators), Kegan Paul, London, 1922.

[30] Wittgenstein, L., Tractatus Logico-Philosophicus, Pears, D.F., McGuinness, B.F. (translators), Routledge \& Kegan Paul, London, 1961. 